[0212-7199 (2004) 21: 5; pp 215-222] ANALES DE MEDICINA INTERNA Copyright @ 2004 ARAN EDICIONES, S.L.

An. Med. InTERna (Madrid) Vol. 21: N. ${ }^{\circ}$ 5; pp 215-222, 2004

\title{
Tuberculosis en el área de Santiago de Compostela durante los años 1999, 2000, 2001 y 2002. Un estudio epidemiológico
}

\author{
M. SALGUEIRO RODRÍGUEZ, J. GONZÁLEZ BARCALA, C. ZAMARRÓN SANZ, \\ M. POMBO PASÍN, J. RICOY GABALDÓN, M. B. PRESEDO GARAZO, \\ U. CALVO ÁLVAREZ, M. L. PÉREZ DEL MOLINO'1, J. ANTÚNEZ LÓPEZ², \\ J. L. DURÁN RIVAS ${ }^{3}$, J. R. RODRÍGUEZ SUÁREZ \\ Servicio de Neumología, 'Servicio de Microbiología, ${ }^{2}$ Servicio de Anatomía Patológica, \\ ${ }^{3}$ Laboratorio Central. Hospital Clínico Universitario. Santiago de Compostela
} TUBERCULOSIS IN SANTIAGO DE COMPOSTELA FROM 1999 TO
2002. AN EPIDEMIOLOGICAL STUDY

\section{RESUMEN}

Objetivo: conocer los parámetros epidemiológicos entre los años 1999 y 2002 de la enfermedad tuberculosa (TB) en nuestra área, que consta de 386.125 habitantes.

Métodos: se consideró caso TB el que cumplía los siguientes criterios: 1) hallazgos microbiológicos y/o patológicos diagnósticos de TB, en cualquier espécimen, 2) pacientes menores de 35 años con historia clínica reciente compatible con TB, con test de Mantoux significativo, linfocitosis y adenosina deaminasa (ADA) en líquido pleural $>47 \mathrm{UI} / \mathrm{mL}$.

Resultados: Hemos estudiado 946 pacientes (568 hombres y 378 mujeres), con extremos de 2 meses y 96 años. El número de casos fue de 235 en 1999, 261 en 2000, 239 en 2001 y 211 en 2002. La incidencia de TB fue de 60,9 en 1999, de 67,6 en 2000, de 61,9 en 2001 y de 54,6 en 2002 , con una incidencia de formas contagiosas de 37,6 en 1999, de 42,5 en 2000, de 37,3 en 2001 y de 31,1 en 2002. La incidencia de meningitis tuberculosa fue de 1,03 en 1999 y 2000, 0,77 en 2001 y 0,51 en 2002. Las formas de asociación con VIH fue de 3,4\% en 1999, de 1,9\% en $2000,2,4 \%$ en 2001 y 2002 . La relación hombre/mujer fue de 1,37 en $1999,1,46$ en $2000,1,52$ en 2001 y 1,74 en 2002. En tasas absolutas, el $38,1 \%$ de ambos sexos se encontraban entre 15 y 34 años y el $49,8 \%$ entre 15 y 44 años, aquí con predominio femenino. Se evidenció un importante aumento en mayores de 55 años, con predominio masculino, tanto en valores absolutos como relativos, con incidencias de 122,4 en $1999,142,8$ en 2000,115 en 2001 y 119 en 2002 , mientras que en mujeres fue de 40,6 en 1999, 60,9 en 2000, 54,1 en 2001 y 39,1 en 2002.

Conclusiones: La incidencia de la tuberculosis en nuestro medio ha disminuido en los últimos años, especialmente en el último, pero se mantiene elevada en los varones mayores de 55 años.

PALABRAS CLAVE: Tuberculosis. Epidemiología. Incidencia. Meningitis tuberculosa.

\section{ABSTRACT}

Objective: The purpose of this study was to determine the epidemiological characteristics of tuberculosis (TB) in the Public Health System District of Santiago de Compostela (population : 386,125) from 1999 to 2002.

Methods: Inclusion criteria were: 1) microbiological and/or pathological diagnosis of TB in any specimen, and 2) patient younger 35 years old with recent medical history of TB. Mantoux test positive, and pleural effusion with linfocitosis and adenosine deaminase $>47 \mathrm{IU} / \mathrm{ml}$.

Results: 946 patients were included (568 men and 378 women), with ages ranging from 2 months to 96 years. The incidence of TB was 60.9/100,000 in 1999, 67.6/100,000 in 2000, 61.9/100,000 in 2001 and $54.6 / 100,000$ in 2002. The incidence rate of tuberculous meningitis was 1.03/100,000 in 1999 and 2000, 0.77/100,000 in 2001 and 0.51/100,000 in 2002. The percentage of cases associated with HIV was $3.4 \%$ in 1999 , $1.9 \%$ in 2000, 2.4\% in 2001 and 2002. We found an increase in the rate of males over 55 years of age; with incidence per 100,000 inhabitants of 122.4 in 1999, 142.8in 2000, 115 in 2001 and 119 in 2002, whereas in females the incidence was 40.6 in 1999, 60.9 in 2000, 54.1 in 2001 and 39.1 in 2002.

Conclusions: In last four years the incidence of tuberculosis has decreased but remains high in males over 55 years old.

KEY WORDS: Tuberculosis. Epidemiology. Incidence. Tuberculous meningitis.

Salgueiro Rodríguez, M, González Barcala J, Zamarrón Sanz C, Pombo Pasín M, Ricoy Gabaldón J, Presedo Garazo MB, Calvo Álvarez U, Pérez del Molino ML, Antúnez López J, Durán Rivas JL, Rodríguez Suárez. JR. Tuberculosis en el área de Santiago de Compostela durante los años 1999, 2000, 2001 y 2002. Un estudio epidemiológico. An Med Interna (Madrid) 2004; 21: 215-222.

\section{INTRODUCCIÓN}

Tras un primer intento de averiguar la magnitud de la enfermedad tuberculosa en nuestro entorno, no fue sino hasta
1993 (1), 1996 (2) y 1998 (3), cuando conseguimos obtener datos epidemiológicos fidedignos mínimos, porque, al igual que en los anteriores estudios, seguimos sin poder constatar los casos diagnosticados en la medicina privada.

Trabajo aceptado: 10 de diciembre de 2003

Correspondencia: Marcelino Salgueiro Rodríguez. Calle San Pedro de Mezonzo, 39, $8^{\circ}$ B. 15701 Santiago de Compostela. A Coruña 
En el presente estudio, una vez más, hemos investigado la situación actual de la enfermedad durante los años 1999, 2000, 2001 y 2002, que, según datos demográficos del Padrón de 2001, incluye una población de 385.364 habitantes.

\section{MATERIAL Y MÉTODOS}

Se llevó a cabo una búsqueda activa y exhaustiva de los casos de tuberculosis (TB) suministrados por los Servicios de Neumología, Microbiología, Anatomía Patológica y Laboratorio Central del Hospital Clínico Universitario de Santiago de Compostela.

Los criterios de inclusión fueron todos los casos que cumplían alguno de los siguientes criterios: A) visión directa del bacilo de Koch mediante tinción de Ziehl-Nielsen (Z-N) o auramina en cualquier espécimen, B) Cultivo positivo para Mycobacterium tuberculosis en medio de Coletsos y/o Middlebrook K-7H9 (C-M) también en cualquier espécimen, C) informe anátomo-patológico con descripción de granulomas y necrosis caseosa (aún sin tinción ni visión de bacilos de Koch) en cualquier muestra de tejido, D) pacientes menores de 35 años con historia clínica compatible y derrame pleural con Mantoux significativo ( $>5 \mathrm{~mm}$ induración en el lugar de la inyección intradérmica en la cara palmar del antebrazo de 2 TU PPD y lectura a las 48-72 horas), linfocitosis en el sedimento pleural y cifras de adenosin-deaminasa (ADA) iguales o mayores de $47 \mathrm{UI} / \mathrm{ml}$ en líquido pleural (5-8), E) los casos de meningitis TB se diagnosticaron por la clínica o cultivo positivo para bacilo de Koch en medio de C-M en el líquido cefalorraquídeo. municipios correspondientes al Área Sanitaria de las provincias de La Coruña y Pontevedra.

No se han incluido en este estudio casos con diagóstico de sospecha clínica ni con tratamiento "ad juvantibus", excepto las TB pleurales con los criterios indicados.

El ámbito territorial de la población incluida en este estudio es la misma que en los anteriores (2-4), que se correspondían administrativamente al Área Sanitaria de Santiago, que actualmente se denomina Área de Atención Primaria de Santiago a la que hemos añadido los municipios de Valga y Pontecesures de la provincia de Pontevedra, ahora segregados. Según el censo de 2001 dicha población es de 385.364 habitantes, cuando era de 392.000 habitantes en 1991 y de 378.000 habitantes en 1995.

\section{RESULTADOS}

Hemos estudiado un total de 946 pacientes (568 hombres y 378 mujeres), con extremos de 2 meses y 96 años. El número de casos fue de 235 (Edad media \pm Desviación Estándar :

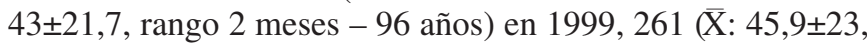
rango 3 meses - 94 años) en 2000, 239 (X): 42,7 $\pm 23,6$, rango

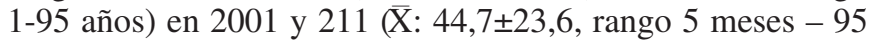
años) en 2002. (Tabla I).

En la tabla II se expresan los datos de incidencia anual, que ha sido de 59,7/100.000 en $1999,66,7 / 100.000$ en 2000, $61 / 100.000$ en 2001 y 52,9/100.000 en 2001, siendo la incidencia de formas transmisibles de 37,6/100.000 en 1999, $42,5 / 100.000$ en $2000,37,3 / 100.000$ en 2001 y $31,1 / 100.000$ en 2002.

TABLA I

DATOS ESTADÍSTICOSS DE LA POBLACIÓN TUBERCULOSA ESTUDIADA DURANTE LOS AÑOS 1999, 2000, 2001 Y 2002 EN EL ÁREA DE SANTIAGO DE COMPOSTELA

\begin{tabular}{lcccc}
\hline Años & 1999 & 2000 & 2001 & 2002 \\
\hline $\bar{X}(a) \pm$ DE & $44 \pm 21,7$ & $45,9 \pm 23$ & $42,9 \pm 23,6$ & $44,7 \pm 23,6$ \\
Rango & $2 \mathrm{~m}-96 \mathrm{a}$ & $3 \mathrm{~m}-94 \mathrm{a}$ & $1 \mathrm{a}-95 \mathrm{a}$ & $5 \mathrm{~m}-95 \mathrm{a}$ \\
Relación H/M & 1,37 & 1,56 & 1,44 & 1,64 \\
Totales (n) & 235 & 261 & 239 & 211 \\
\hline
\end{tabular}

$\mathrm{X}=$ media en años; $\mathrm{m}=$ meses; $\mathrm{a}=$ años; $\mathrm{DE}=$ desviación estándar; $\mathrm{H}=$ hombre; $M=$ mujer; $\mathrm{n}=$ número de casos

Con los datos obtenidos hemos obtenido los indicadores epidemiológicos siguientes: 1) tasa de incidencia anual de la TB por 100.000 habitantes (/100.000), 2) incidencia /100.000 de formas transmisibles (definidas como nuevos casos de la enfermedad con baciloscopia positiva y/o cultivo en medio de $\mathrm{C}-\mathrm{M}$ positivo en cualquier muestra bronquial, aspirado gástrico en ayunas y/o el diagnóstico anátomo-patológico ya considerado en muestras procedentes de vías aéreas), 3) incidencia de meningitis TB/100.000, 4) relación Hombre/Mujer (H/M), 5) porcentaje de casos con co-infección con $\mathrm{VIH}, 6)$ formas clínicas de presentación en porcentaje y 7) número de casos e incidencias relativas $/ 100.000$, por grupos de edad y sexo degún los datos por sexo y grupos quinquenales de edad en los
La incidencia de meningitis TB se expone en la Tabla III, destacando un caso menor de 5 años en el año 2000 y otro en el 2001.

La asociación con VIH + se expone en la tabla IV.

En la tabla $\mathrm{V}$ vemos las diversas formas de presentación de la enfermedad, dónde destacamos un progresivo incremento de las formas pleurales.

El total de casos y sus porcentajes se resume en la tabla 6 , en la que observamos que el 38,1\% tiene menos de 34 años y el $49,8 \%$ menos de 44 años, siendo precisamente en esta población donde se encuentra la mayoría de las formas transmisibles. En esta tabla se aprecia también un predominio femenino de casos en la población entre 15 y 34 años. 
TABLA II

INCIDENCIA DE LA ENFERMEDAD Y FORMAS CONTAGIOSAS (X 100.000 H) DE CASOS DE TUBERCULOSIS DURANTE LOS AÑOS 1999, 2000, 2001 Y 2002 EN EL ÁREA DE SANTIAGO DE COMPOSTELA (POBLACIÓN 386.125 H)

\begin{tabular}{lcccc}
\hline Años & 1999 & 2000 & 2001 & 2002 \\
\hline $\begin{array}{l}\text { Incidencia } \\
(\mathrm{X} 100.000 \mathrm{H})\end{array}$ & 60,9 & 67,6 & 61,9 & 54,6 \\
$\begin{array}{l}\text { Totales }(\mathrm{n}) \\
(\mathrm{BK}+\mathrm{X} 1000.000 \mathrm{H})\end{array}$ & 37,6 & 42,5 & 239 & 211 \\
\hline
\end{tabular}

$\mathrm{H}=$ habitantes; $\mathrm{BK}+=$ baciloscopias positivas

TABLA III

CASOS DE MENINGITIS TUBERCULOSA E INCIDENCIA (X 100.000H) DURANTE LOS AÑOS 1999, 2000, 2001 Y 2002 EN EL ÁREA DE SANTIAGO DE COMPOSTELA

\begin{tabular}{lcccc}
\hline Años & 1999 & 2000 & 2001 & 2002 \\
\hline Hombres & 1 & 2 & 1 & 1 \\
Mujeres & 3 & 2 & 2 & 1 \\
$>$ de 5 años & 0 & 1 (varón) & 1 (varón) & 0 \\
Totales & 4 & 4 & 3 & 2 \\
Incidencia $(\mathrm{X} 100.000 \mathrm{H})$ & 1,03 & 1,03 & 0,77 & 0,51 \\
\hline
\end{tabular}

TABLA IV

NÚMERO DE CASOS Y PORCENTAJES DE LA ASOCIACIÓN TUBERCULOSIS Y VIH+ DURANTE LOS AÑOS 1999, 2000,2001 Y 2002 EN EL ÁREA DE SANTIAGO DE COMPOSTELA

\begin{tabular}{lcccc}
\hline Años & 1999 & 2000 & 2001 & 2002 \\
\hline Hombres & 8 & 4 & 5 & 3 \\
Mujeres & 0 & 1 & 1 & 2 \\
Totales & 8 & 5 & 6 & 5 \\
Porcentaje & 3,4 & 1,9 & 2,4 & 2,4 \\
\hline
\end{tabular}

En las figuras 1 a 4 se expresan el número de casos y la incidencia relativa para cada grupo de edad y sexo. Es de señalar una alta incidencia relativa en sujetos varones a partir de 55 años, donde se presenta con tasas hasta 2 veces superiores a las media del total de la población y de 2 a 4,9 veces por encima del mismo grupo de edad de mujeres, como sucede en el años 2002 en las poblaciones del grupo 55-64 años. También, en relación con este hallazgo, se observa una tendencia a aumentar desde 1999 a 2002.

\section{DISCUSIÓN}

Ante los datos actuales, en comparación con los 10 años previos, (1-3), es evidente que las tasas de incidencia anual y de formas transmisibles muestran un ligero descenso, especialmente en el último año, similares a otros estudios de nues- tra comunidad $(6,7)$, aunque la incidencia se encuentre todavía más elevada que la de nuestro entorno $(8,11)$, excepto el norte de Portugal (12). En este punto, ante la literatura revisada $(13,14)$, teniendo en cuenta de que partíamos de cifras mucho más altas, parece evidente que en nuestra población comenzamos ahora a tener resultados favorables.

Persiste, sin embargo, la alta incidencia de la enfermedad en edades jóvenes (considerando jóvenes a los de edad igual o menor de 44 años), tal como ocurre en los países en vías en vías de desarrollo $(15,16)$, con predominio de mujeres, igual que ocurría hace años entre la población de color en los Estados Unidos (17).

Resulta de difícil explicación que nuestros indicadores epidemiológicos permanezcan en niveles que consideramos demasiado elevados, sobre todo si consideramos: a) las cifras en comparación con otras comunidades ibéricas (6-14); b) la baja resistencia a fármacos tanto primaria como secundaria (18-20); 
TABLA $\vee$

FORMAS DE PRESENTACIÓN DE LA TUBERCULOSIS EN LA POBLACIÓN ESTUDIADA, EXPRESADA EN PORCENTAJES, DURANTE LOS AÑOS 1999, 2000, 2001 Y 2002 EN EL ÁREA DE SANTIAGO DE COMPOSTELA

\begin{tabular}{lcccc}
\hline Formas de presentación & 1999 & 2000 & 2001 & 2002 \\
\hline Pulmonar (\%) & 61,7 & 62,8 & 60,3 & 56,9 \\
Pleural (\%) & 19,6 & 23 & 22,6 & 27 \\
Ganglional (\%) & 10,6 & 6,5 & 13,8 & 6,6 \\
Miliar (\%) & 1,7 & 2,3 & 1,8 & 1,4 \\
Meníngea (\%) & 1,7 & 1,9 & 1,3 & 0,9 \\
Otras (\%) & 14,8 & 13,4 & 13 & $15,6(*)$ \\
\hline
\end{tabular}

${ }^{*}$ ) Un caso de Micobacterium Avirum Intracelulare

TABLA VI

RESUMEN DEL TOTAL DE CASOS Y PORCENTAJE ENTRE LOS 15 Y LOS 44 AÑOS, POR GRUPOS DE EDAD Y SEXO DE 1999 A 2002

\begin{tabular}{|c|c|c|c|c|c|c|c|c|}
\hline Edad (años) & 15 & 34 & Total 15-34 & 35 & -44 & Total & $15-44$ & Totales \\
\hline Sexo & $\mathrm{H}$ & $M$ & $\mathrm{H}+\mathrm{M}$ & $\mathrm{H}$ & $M$ & $\mathrm{H}$ & $M$ & $\mathrm{H}+\mathrm{M}$ \\
\hline Número de casos & 178 & 187 & 365 & 75 & 37 & 253 & 224 & 477 \\
\hline Total & 18,6 & 19,5 & $38,1 \%$ & 7,8 & 3,9 & 26,4 & 23,4 & $49,8 \%$ \\
\hline
\end{tabular}

$\mathrm{H}=$ hombres; $M=$ mujeres

c) el programa gallego de lucha y prevención de la tuberculosis que incluye vigilancia epidemiológica activa y tratamiento supervisado, directamente observado y curación certificada $(21,22)$; y d) la potente farmacopea disponible, con el amplio uso de fármacos, cuya alternativa en el tratamiento de la tuberculosis goza ya de amplia literatura $(22,23)$. En este punto hemos de llamar la atención sobre el retraso diagnóstico de la enfermedad - hecho evidente en nuestra práctica clínica diaria, imputable al enfermo, al sistema de salud, o a ambos -, de tal forma que con frecuencia la tuberculosis se presenta inicialmente con enfermedad extensa y cavitada, y se sabe que la formación de cavernas requiere al menos dos o tres meses, siendo precisamente estas formas cavitadas las que más bacilos diseminan.

Como ya nos hemos pronunciado, en relación con el "modus vivendi" de la población joven (hábitos, conductas y lugares de reunión) y otros factores involucrados en grandes o pequeñas superficies (ocio, industria, comercio), que pueden jugar un importante papel en la transmisión de la enfermedad (25-32), sugerimos que se tengan en cuenta estos factores a la hora de diseñar programas de prevención y lucha, así como induciendo a que se dicten leyes sobre actuaciones preventivas, tales como filtros y radiaciones ultravioleta, cuya utilidad está bien demostrada (33-35).

Así mismo, señalar que se han presentado dos casos de meningitis en niños menores de 5 años, uno en el 2000 y otro en el 2001, lo cual, desde el punto de vista de control del enfermedad, en visión de Styblo (15), se considera un indicador de alerta.
Tras las consideraciones apuntadas y ante la literatura revisada, aún hemos de discutir otros hechos no fáciles de explicar. Tal como ya hemos comunicado anteriormente (1-3) $\mathrm{y}$ al igual que otros autores en la población gallega $(6,36)$, seguimos encontrando un bajo porcentaje de la asociación VIH/tuberculosis. Por ello - al contrario que en la publicación ya clásica en la ciudad de Nueva York (37), y otras en las que España figura con los porcentajes más altos de Europa de SIDA y de coinfección VIH/tuberculosis (13-19) -, objetivamos que el advenimiento del retrovirus no parece haber tenido una incidencia significativa tanto en Galicia como en nuestra área de influencia, con un perfil francamente descendente en los últimos años (38).

Pero, ante los resultado de este estudio, lo que más nos preocupa es la población masculina de mayores de 55 años, dónde al expresar los datos en incidencia específica, nos llaman la atención las elevadas tasas de incidencia, más del doble por encima de la media, con valores que sobrepasan la tasa de 100/100.000 con tendencia a incrementarse, y de 2 a 4,9 veces superiores a las de la población femenina de la misma edad. Es precisamente en ésta población dónde la enfermedad debuta con presentaciones atípicas, de insospechado diagnóstico, que hacen bueno el adagio que califica a la tuberculosis como "la gran simuladora", tanto según nuestra apreciación (39), como la de otros autores $(40,41)$ - con especial referencia a los trabajos de Zamarrón y cols. (42) y Lado y cols. (43), ya que sus datos están referidos a la población de esta misma área sanitaria -. Permanecen, pues, muchas incóg- 


$$
\text { Hombres Mujeres } \square \text { Hombres/100.000 } \square \text { Mujeres/100.000 }
$$

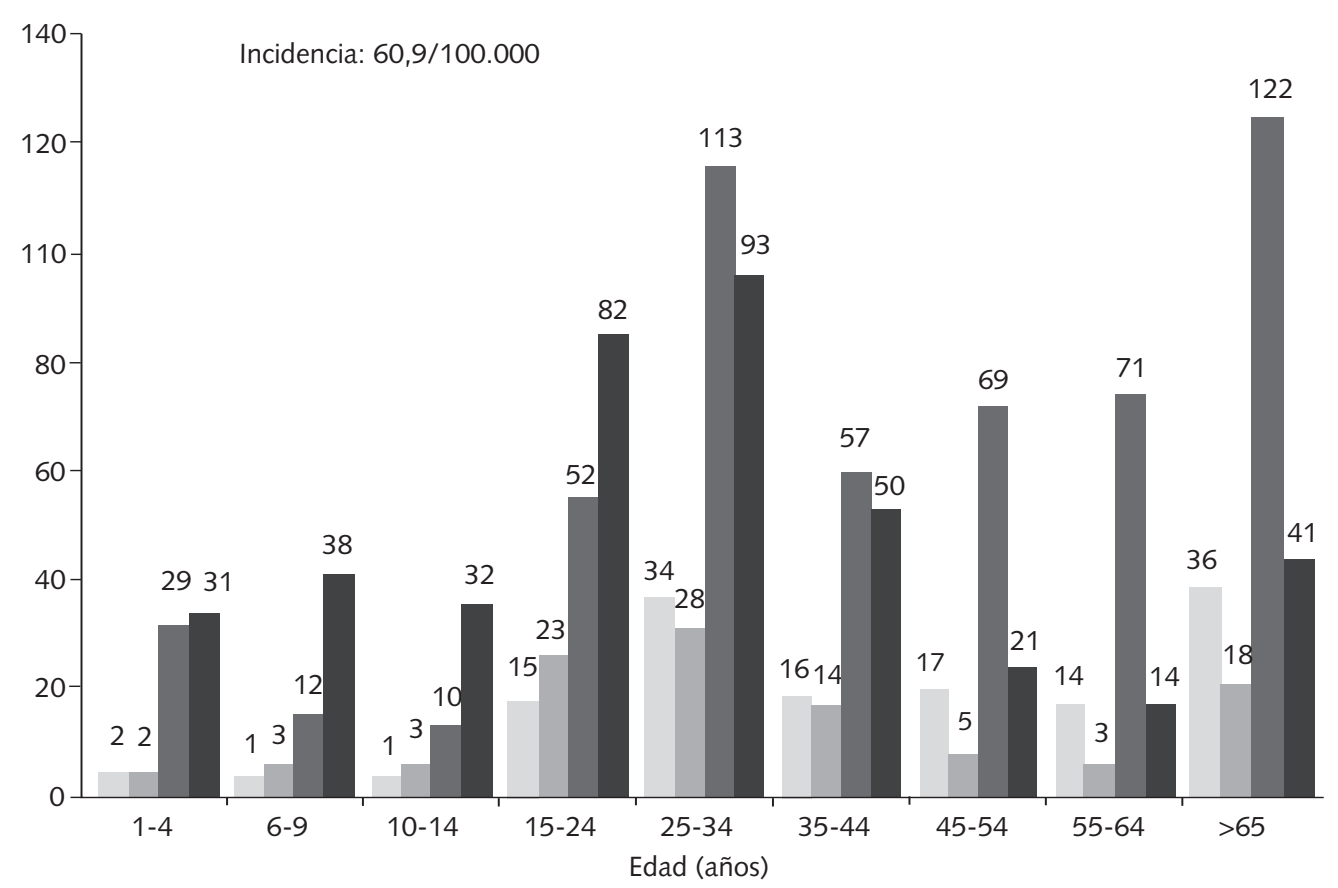

Fig. 1. Número de casos e incidencia relativa, por 100.000 habitantes (/100.000), por grupos de edad y sexo, en la población estudiada. Sobre el gráfico se expresa la incidencia. Año 1999.

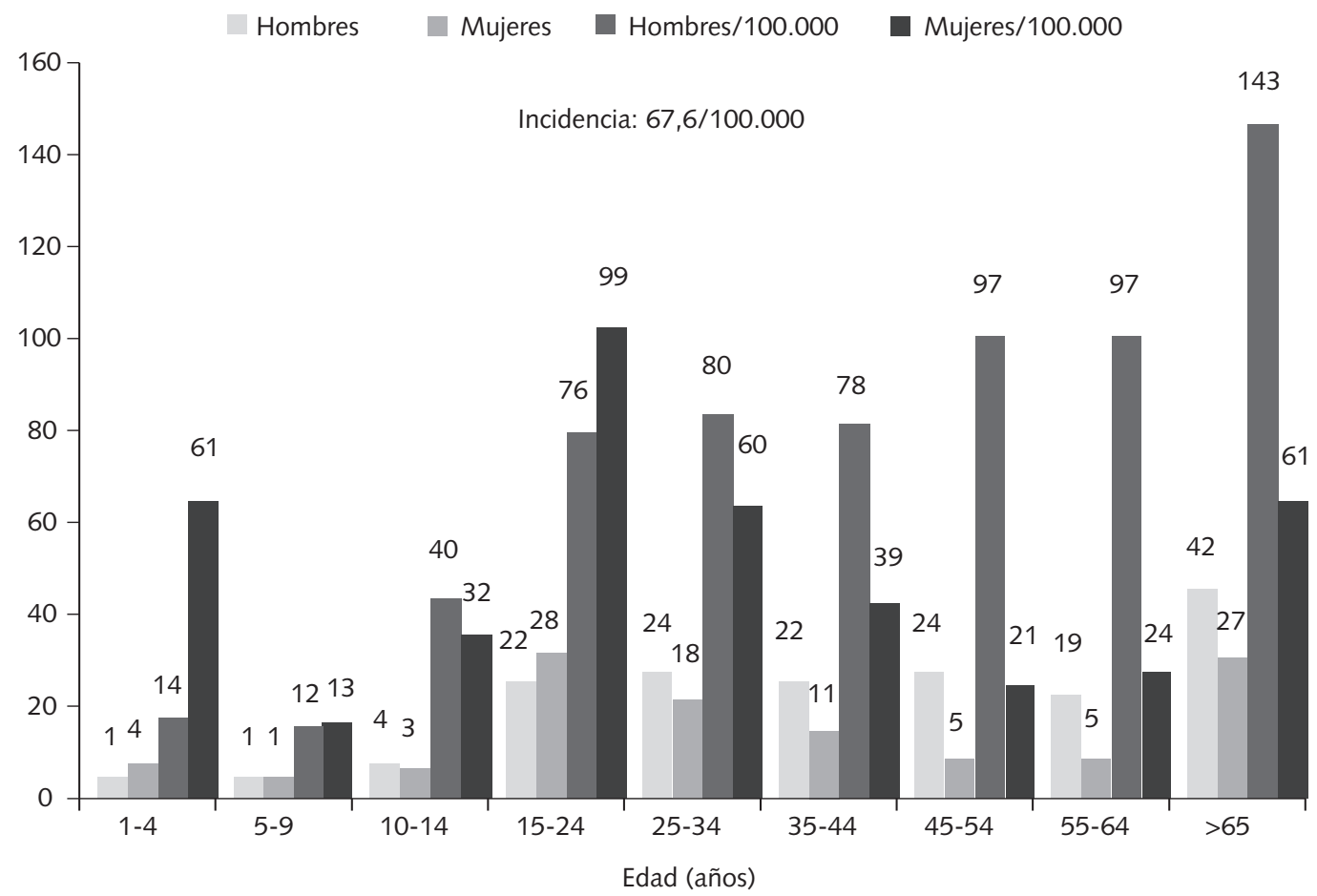

Fig. 2. Número de casos e incidencia relativa, por 100.000 habitantes (/100.000), por grupos de edad y sexo, en la población estudiada. Sobre el gráfico se expresa la incidencia. Año 2000. 
Hombres Mujeres $\square$ Hombres/100.000 Mujeres/100.000

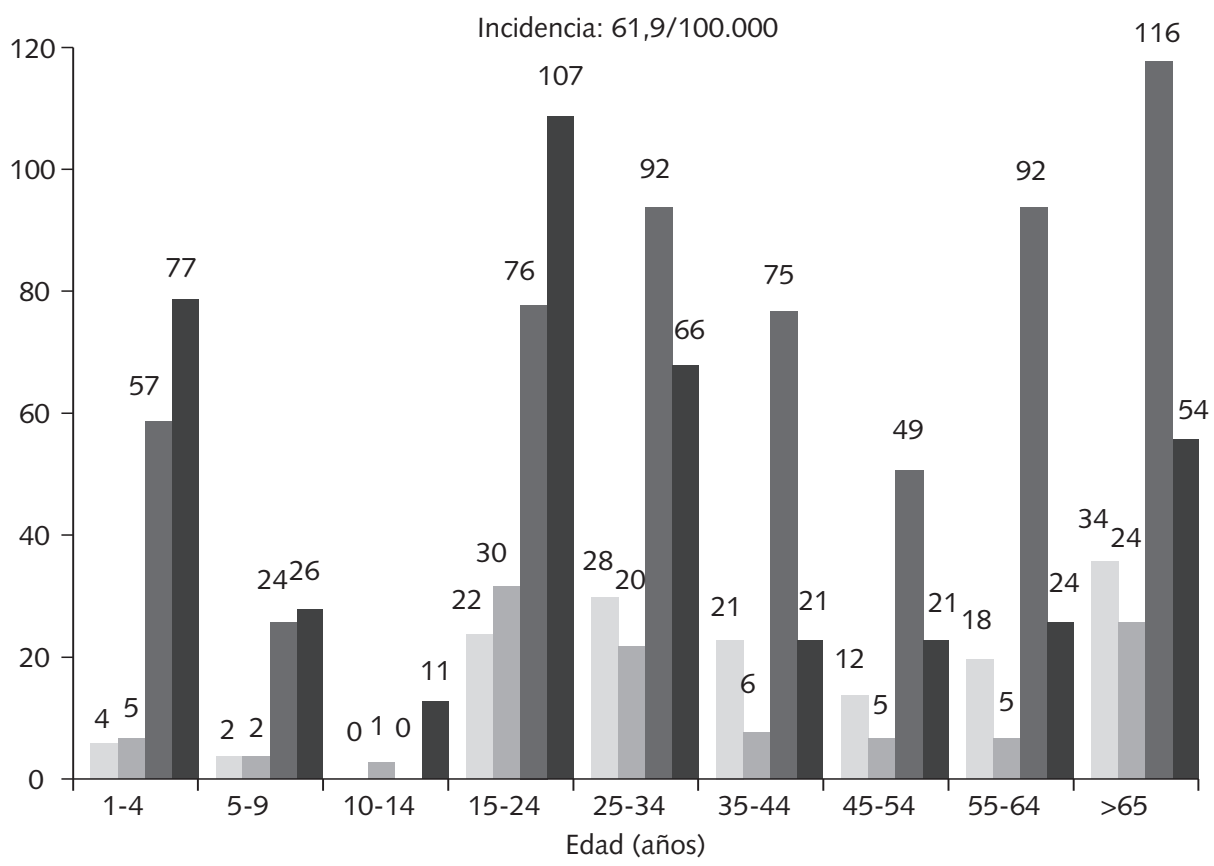

Fig. 3. Número de casos e incidencia relativa, por 100.000 habitantes (/100.000), por grupos de edad y sexo, en la población estudiada. Sobre el gráfico se expresa la incidencia. Año 2001.

Hombres Mujeres $\square$ Hombres/100.000 $\square$ Mujeres/100.000

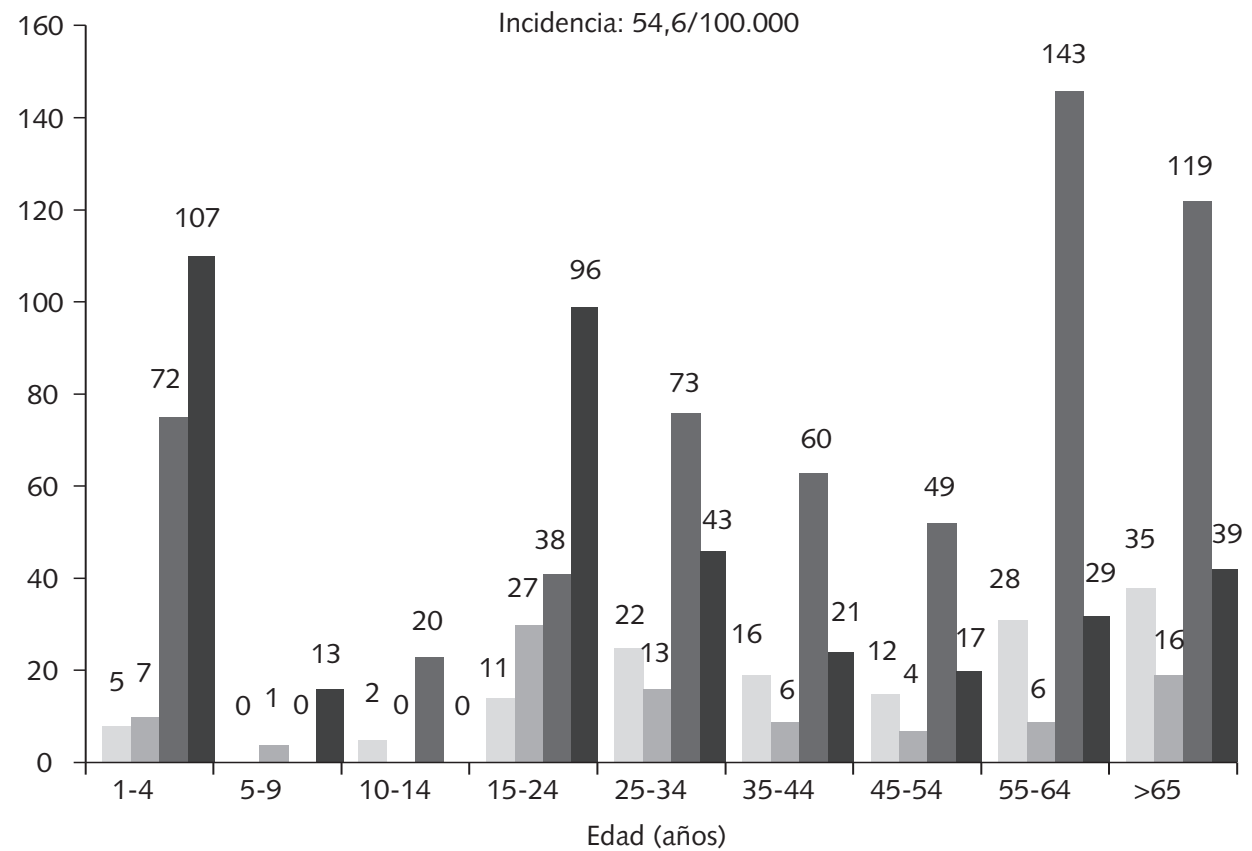

Fig. 4. Número de casos e incidencia relativa, por 100.000 habitantes (/100.000), por grupos de edad y sexo, en la población estudiada. Sobre el gráfico se expresa la incidencia. Año 2002. 
nitas médicas y epidemiológicas en relación con esta población masculina, que siendo mucho menos numerosa que la femenina, presenta una incidencia que llega a ser el doble o hasta cerca de cinco veces más alta. Por último otro punto que creemos fundamental es llegar a saber si la enfermedad en nuestros ancianos debuta como caso nuevo o si se debe a casos antiguos reactivados. Por ello, además del despistaje de contactos (44) y estudios socio-económicos, demográficos y del entorno familiar, tal como sugieren en el norte de Portugal, donde la tasa de incidencia, es similar a la nuestra $(12,45)$, serían primordiales estudios genéticos y de epidemiología molecular (46-48), puesto que, lo que hasta hace poco significaba una utopía, actualmente está al alcance de varios laboratorios del país.

Con todo, habría que seguir valorarando los viejos factores, siempre citados, de hacinamiento, mal nutrición y pobre- za, que podrían haber regresado y volver a jugar un nuevo e impensado papel.

En conclusión, en nuestra área sanitaria la tuberculosis permanece endémica, con tendencia al descenso especialmente en el último año y que el ser varón mayor de 55 años representa un factor de riego para padecer tuberculosis al menos el doble mayor que el resto de la población.

\section{AGRADECIMIENTOS}

Al Dr. Albes de Pediatría, al Dr. Brea de Codificación, a la Dra. Fondevila del Archivo Central, a la Dra. Losada de Medicina Interna, a Pablo Durán Pérez estudiante de Medicina y al Sr. Maneiro de Administración, por su inestimable colaboración.

\section{Bibliografía}

1. Salgueiro M, Zamarrón C, Alvarez-Calderón P, Tumbeiro M, Penela P, Valle JM et al. Estudio epidemiológico de la tuberculosis en el Área Sanitaria de Santiago de Compostela durante los años 1989, 1990 y 1991. An Med Interna (Madrid) 1993; 10: 427-32.

2. Salgueiro M, Zamarrón C, Otero Y, Alvarez-Dobaño JM, Arias MR, Alvarez-Calderón P, et al. Estudio epidemiológico de la tuberculosis en el Área Sanitaria de Santiago de Compostela durante los años 1992, 1993 y 1994. An Med Interna (Madrid) 1996; 13: 111-4.

3. Salgueiro Rodríguez M, Zamarrón Sanz C, González Barcala J, Vilas Iglesias A, Suárez Antelo J, Durán Rivas JL, et al. Estudio epidemiológico de la tuberculosis en el Área Sanitaria de Santiago de Compostela durante los años 1995, 1996, 1997 y 1998. An Med Interna (Madrid) 2001; 18: 20-3.

4. Valdés L, Alvarez D, San José E, Juanatey JRG, Valle JM, Salgueiro M, et al. Value of Adenosine deaminase in the diagnosis of tuberculous pleural effusions in young patients in a region of high prevalence of tuberculosis. Thorax 1995; 50: 600-3.

5. Light RW, Ferrer J. Diagnóstico de la pleuritis tuberculosa. Arc Bronconeumol 1999; 35: 105-7.

6. Anibarro García R, Vázquez-Gallardo R, Toubes Navarro ME, Penas Truque A, Lema Mougán R, Túñez Bastida V, et al. Epidemiología de la tuberculosis en Galicia. An Med Interna (Madrid) 1999; 16: 290-6.

7. Díez Ruiz-Navarro M, Hernández Espinosa JA, Coloto González T, Castells Carrillo C, Domínguez García A, et al. Resultados del tratamiento antituberculoso en seis regiones autonómicas de España. Med Clin (Barcelona) 2001; 117: 574-80.

8. Caminal Montero L, Trapiella Martínez L, Telenti Asensio M, Fernández Bernaldo de Quirós J. Caraterísticas de la tuberculosis en un hospital general durante el período 1993-1998. Análisis de la resistencia y de la coinfección VIH. Enferm Infecc Microbiol Clin 2002; 20: 68-73.

9. Martín V, Aránzazu Alonso M, Ramos J, Otero A, Cortizo J, Travieso $\mathrm{S}$. La incidencia de la tuberculosis respiratoria en la provincia de León de acuerdo con el sistema de notificación de enfermedades de declaración obligatoria, 1992-1999. Rev Esp Salud Pública. 2002; 76: 239-48.

10. Iglesias Gozalo MJ, Rabanaque Hernández MJ, Gómez López LI. Tuberculosis en la provincia de Zaragoza. Estimación mediante el método captura-recaptura. Rev Clin Esp 2002; 202: 249-54.

11. Campos Rodríguez F, Muñoz Lucena F, Umbría Domínguez S, Reyes Núñez N, De la Cruz Morón I, Nogales Pérez MC. Tendencias en la incidencia de la tuberculosis en el área de Sevilla - Sur de España durante los años 1990. Arch Bronconeumol 2002; 38: 214-20.

12. Workshop comemorativo do Día Mundial da Tuberculose. Boletim Da Sociedade Portuguesa de Pneumologia. 2003; 47-48: 3-13.

13. Puerto Alonso JL, García-Martos P, Marín Casanova P, Saldarreaga Marín A, Vega Elías J de L, Mira Gutiérrez J. Valoración de la aplicación de un programa de prevención y control de tuberculosis. Enferm Infecc Microbiol Clin 2002; 20: 150-3.

14. Caylá JA, Jansá JM, Galdós-Tangüis H. 4. Prevención y control de la tuberculosis en las grandes urbes, en J. Sauret Valet. Tuberculosis. Visión Actual. Ed Aula Médica S.A. Madrid 2001: 71-94.

15. Styblo K. Overview and epidemiologic assessment of the current global tuberculosis situation with an emphasis on control in developing countries. Rev Infect Dis 1989; 11: 339-46.

16. Murray CJL, Styblo K, Rouillon A. Tuberculosis in developing countries: burden, intervention and cost. Bull Int Union Tuberc 1990; 65: 120 .

17. Center for Disease Control. Tuberculosis in the United States, 1978. HHS Publication No (CDC) 80-8322 Atlanta: Center for Disease Control 1980. p. 3.

18. Idígoras P, Valiente A, Iglesias L, Piñeiro L, Pérez-Trallero E. Bajos niveles de resistence a drogas en muestras de Mycobacterium tuberculosis aisladas en Guipuzkoa (1993-2001). Enferm Infecc Microbiol Clin 2002; 20: 187-8.

19. Alvarez D, Valle JM, Martino MV, Gordo P, Carreira J, Valdés L. Estudio de las resistencia primarias a fármacos antituberculosos en Galicia. Arch Bronconeumol 1994; 30: 297-300.

20. Pérez del Molino, ML, Pardo F, Carballo E, Gutiérrez MC. Resistencia de Mycobacterium tuberculosis a fármacos en un área de tuberculosis endémica. Rev Esp Quimioterap 1994; 7: 137-41.

21. Weis SW. Universal Directely observed therapy. A treatment for tuberculosis. Clinincs in Chest Medicine 1997; 18: 155-63.

22. American Thoracic Society Documents. American Thoracic Society/ Centers for disease control and prevention/ Infectious Diseases Society of America: treatment of tuberculosis. Am J Respir Crit Care Med 2003; 167: 603-62.

23. Gillespie SH, Gosling RD, Uiso LO, Bongard E, Kanduma EG, Nyindo $\mathrm{M}$, et al. The bactericidal activity of moxifloxacin in patients with pulmonary tuberculosis. www.AJRCCM.ORG; 13-agosto-2003.

24. Kline SE, Hedemark LL, Davies SF. Oubreak of tuberculosis among regular patrons of a neghborhood bar. E Engl J Med 1995; 333: 222-7.

25. Dutt AK, Mehta JB, Whitaker BJ, Westmoreland H. Oubreak of tuberculosis in a church. Chest 1995; 107: 447-52.

26. Stead WW, Lofgren JP, Warren E, Thomas C. Tuberculosis as an endemic and nosocomial intection among elderly in nursing homes. N Engl J Med 1985; 312: 1483-87.

27. Martín V, Caylá JA, Bolea A, De Paz JA. Evolución de la prevalencia de la infección por Mycobacterium Tuberculosis en una población reclusa al ingreso en prisión entre 1991 y 1996. Med Clin (Barc) 1998 111-11-6.

28. Sánchez Moreno A, Villena L, Castro JM, Aguayo E, Pujol E. Estudio de tuberculosis en la prisión de Huelva. An Med Intern (Madrid) 1997; 14: $607-10$.

29. Kantor HS, Poblete R, Pusateri S. Nosocomial transmission of tuberculosis from unsuspected disease. Am J Med 1984; 84: 833-8.

30. Ochs LCW. Epidemiology of tuberculosis. JAMA 1962; 179: 247-52.

31. Kenyon Th, Valway S, Ihle W, Onorato I, Castro X. Tansmission of multidrug-resistant Mycobacterum Tuberculosis durin a long airplane flight. N Eng J Med 1996; 334: 933-8.

32. Menzies D, Fanning A, Yuan L, Fitzgerald M. Tuberculosis among health care workers. N Engl J Med 1995; 332: 92-8. 
33. Riley RL, Nardel EA. State of de Art: Clearing the air. The Theory and application of ultraviolet air desinfection. Am Rev Respir Dis 1989; 139: 1286-94.

34. Nardel EA. Environmental control of Tuberculosis. Med Clin N Amer 1993; 77: 1315-34.

35. Smith PG, Mos AR, Chapter 4. Epidemiology of tuberculosis. En Tuberculosis, Protection, and Control. Editor: Barry R. Bloom. ASM Press, Washington 1994: 47-59.

36. Lado Lado FL, Barrio Gómez E, Carballo Arceo E, Cabarcos Ortíz de Barrón A. Tuberculosis e infección por el virus de la inmunodeficiencia humana: manifestaciones clínicas y rendimiento de procedimientos diagnósticos según las distintas formas de localización de la enfermedad. An Med Interna (Madrid) 2000; 17: 27-34

37. Brudney K, Dobkin J. Resurgent tuberculosis in New York City. Human immunodeficiency virus, homelessness, and the decline of tuberculosis control programs. Am Rev Respir Dis 1991; 144: 745-9.

38. Documentos Técnicos de Saúde Pública. VIH/SIDA. Informe do Rexistro Galego da Sida. Xunta de Galicia. Xaneiro 2001.

39. Salgueiro Rodríguez M. Editorial. Tuberculosis en pacientes ancianos. An Med Inter (Madrid) 2002; 19: 107-10.

40. Miller RA. The aging immune system: primer and prospectus. Science 1997; 273: 70-4.
41. Pérez-Guzmán C, Vargas MH, Torres-Cruz A, Villarreal-Velarde H. Does aging modify pulmonary tuberculosis?. A meta-analytical reviw. Chest 1999; 116: 961-7.

42. Zamarrón C, Salgueiro M, Álvarez JM, Otero Y, Rodríguez Suárez JR. Características clínicas de tuberculosis pulmonar en el anciano. An Med Interna (Madrid) 1997; 14: 167-9.

43. Lado Lado FL, Túñez Bastida V, Golpe Gómez AL, Cabarcos Ortíz de Barrón A, Pérez del Molino ML. Tuberculosis en pacientes ancianos. Formas de presentación. An Med Interna (Madrid) 2002; 19: 111-4.

44. Weis S. Contact investigations: how do they nedd to be designed for the 21 st century? Am J Respir Crit Care Med 2002; 166: 1016-17.

45. Duarte R. Editorial. Día Mundia da Tuberculose. Boletim da Sociedade Portuguesa de Pneumologia, 2003; 47-48: 1-2.

46. Coll P, March F. 3. Epidemiología molecular de la tuberculosis. En J. Sauret Valet. Tuberculosis. Visión Actual. Madrid: Ed Aula Médica S.A, 2001; p. 59-70.

47. Tobin MJ. Tuberculosis, lung infections, interstitial lung disease, and Journalogy in AJRCCM 2002. Am J Respir Crit Care Med 2003; 167: 345-55.

48. Weis SE, Pogoda JM, Yang Z, Cave MD, Wallace C, Kelley M, et al. Transmissión dynamics of tuberculosis in Tarrant country, Texas. Am J Respir Crit Care Med 2002; 166: 36-42. 